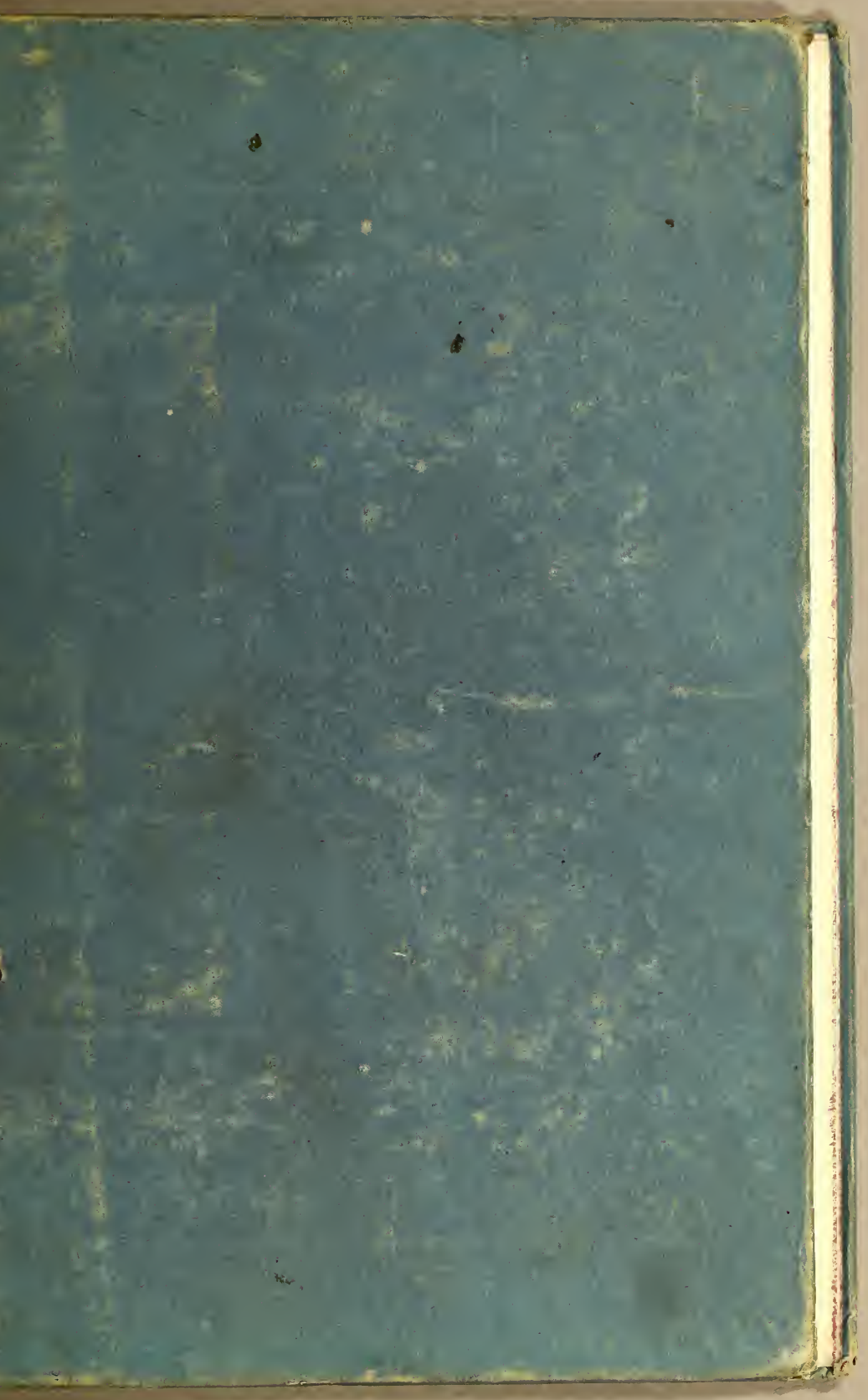




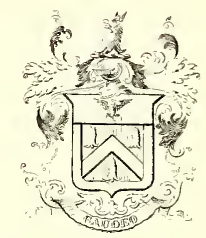

Alaber Carfer 3rome fibrarn

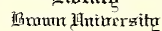




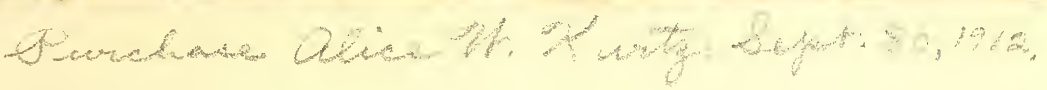




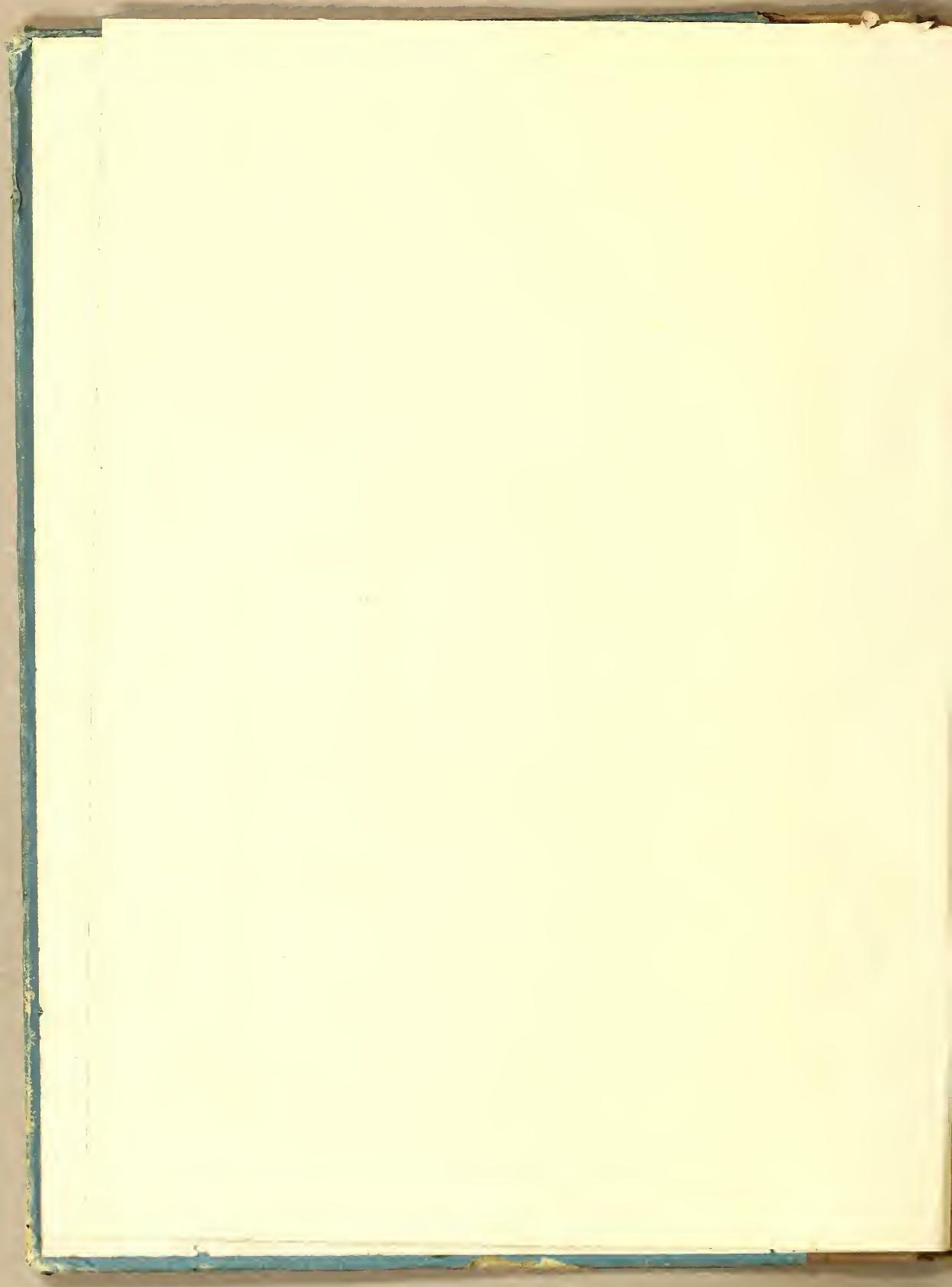




\section{S. D.}

M

Uy Señor mio: Fl amor al Rey à la Patria al bien comuk, à nuesiro propio interes y comodidad, nos conduce à indagar los arcanos de la Naturaleza. Para alabar al Criador, para admirarnos de sa Sabiduria, para sacar alguna utilidad de todo lo que crió para el hombre, que nada hizo ni propagó en vano, y para confundirnos, que de cosas tenemos y hemos tenido despreciadas que e:taban destinadas por el Omniporente para grandes especificos Medicos, para efectos del mas lucratibo comercio, para las fabricas y Artes, ó para los manjares mas deleitosos.

Efectivanente ni el diezmo, y aunque se diga la primicia, de los objetos naturales que nos rodean, sabemos cobrae del derecho que les impuso el Criador en beneficio del hombre. Esto hace ser alabado de rodos y premiado de los Monarcas el que por su aplicacion y talento es autor de algun nutvo des. cubrimisnto, por que redunda en beneficio de todos.

De quantos se hagan en lo succesivo en este Gavinere que se acaba de dar al publico con roda magnificencia (dedicado en su dia al Augusto nombre de nuestra Reyna, de cuyos progresos ha de cuy lat en adelante la Real Sociedad) De todos los descubrimientus que deben esperarse, se llamaràn autores los que con adividad y empeño remiran colecciones de quanto pron duccen sus respectivas Provincias arreglados a la a djunta instruccion,

Este encargo haren à V. la Real Socicdad y el Naturalista de la Real Expedicion, D. Joseph Longinos Martinez en la inteligencia de que verificandolo $V_{0}$ asi no soló acredirarà, que coopera a las Soberanas intenciones del Rey, y al desempeño que en esta parte anelamos nosotros, sino que al mismo riempo, se grangear el conceptode verdadero y celoso de el bien è instrucción del Publi-o, que se interesa tanto en esto.

Dios Guatde á Y. muchos añus, Nucva Guatemala y Encro d. de 1797 . 


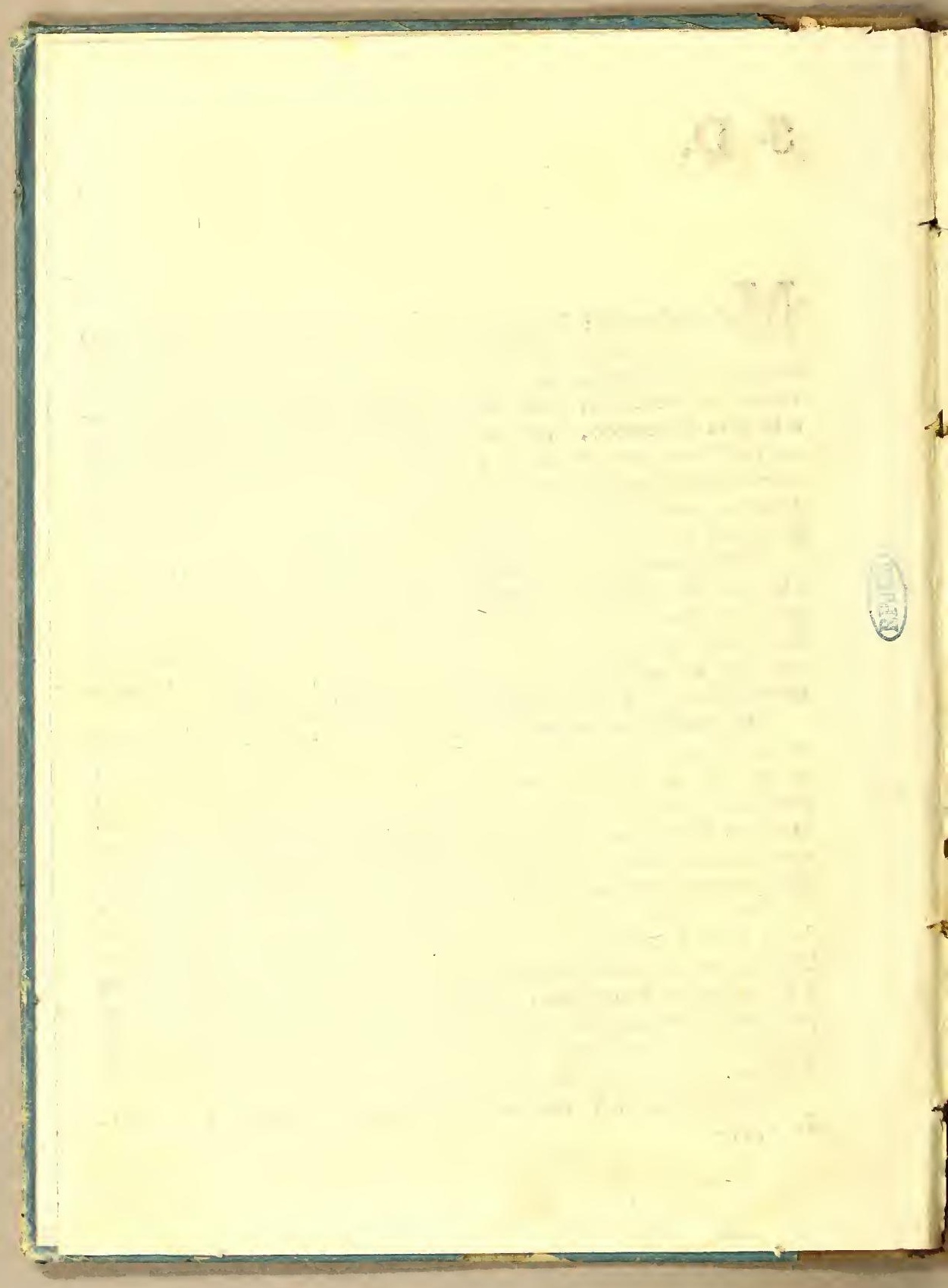




\section{COMPENDIOINSTRUCTIVO}

sobre el modo mas seguro de disponer, juntar, conserbar, y remitir las producciones Naturales, dispuesto por el $\mathrm{Na}$ turalista D. Joseph Longinos Martinez para que sirba de instruccion $y$ acompañe à la adjunta carta Circular.

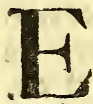

Ste compendio inteligible á roda clase de personas servird de guia a los Socios corresponsales, a los curiosos aman. tes de la Patria, asi Eclesiasticos como secularts, rara que formen sus colecciones, y será muy del caso lo lean algunas veses delante de Sugetos que andan muchas rierras, por que siempre se ronsiguen de estos algunas luces y noticias de producciones que la casualidad suele presentarles.

\section{EN EL RENNO MINERAL}

se colecta lo siguiente.

- As tierras de algun color, las arenas, las arcillas, los
ocres, las tierras blancas y negras, las humosas y calcareas todas las especies de Sales, las piedras de colores, las pre. ciosas, las agatas, cuarzos, o crístales de Rora, jaspes, marmores: toda piedta que tenga alguna transpariencia ò color, duras y blantas, las piedras de $\mathrm{Cal}$, de yeso, de talco, amianro, mica, las variedades de silex, ó federnales, las guijas vetadas y de algun fondo transfarente, toda especie de mina de metal y semimetal, las piritas, las producciones de Volcanes, los betunes, los azufres, las escrias, el Carvon de piedra. \&.c. Las petrificaciones, las concreciomes, las incrustaciones y resijuos de aguas termales y mi cerales. 


\section{(3) \\ DEL RERNU VEGETAL.}

As rayzes tuberosas, ò carnosas, y algunnas de estas leñosas que representan figuras particulares, como las man* dragoras \& e. las de algun uso en la Medicina, industria economia \&c.

Las cortezas balsamicas, ò de algun destino en las manufacturas, comercio, Medicina \&c. Las epidermis, ò telita exterior de los Atboles, de alguna singularidad, ò uso y el liber, ó membrana inmediata à la parte leñosa, que se note en ella alguna rareza, y se le dá algun destino.

La variedad de Maderas de todas especies en pedazos de seis a ocho puigadas de longitud, y tres 6 quatro de diamerro, de ramas de esre grueso que demuestren el corazon, lo restante del léro, y la corteza, con esplicacion del uso que se le dà en sus respectivos parages, à cada una, y del mayor tamaño â que suele llegar cada especie.

Algunos rallos, baras, ó bejucos, que rengan alguna particularidad, ò se haga de ellos algun uso, como las Cañas de Indias que sirven de bastones, otros tallos de Palmas, que se les dè el mismo destino, bejucos que sirben para beber, teñir \&ze.

Las ramitas de todo Arbol y Planta, con for y fruto, extendidas entre papeles sib hay de estraza, y sino del comun, con el cuydado de aprensarlas entre dos tablas, enjugarlas con la miyor prontirus que sea posible poniendolas al sol, y mudantolas papeles secos, $c(n$ el cuydado de conservarlas bien extendidas; y para que les permanezca su color natural, se pasa por el papel, siempre que se mudar, unos polvos, de cal, ò 1 l injre, y en defecto de esros, aunque sea cenizz. Las flores misy singulares se desecan dentro de una vasija entre arena, bien enjuta, mudando ela rodos los dias para asolearla.

Toda especie de semilia se seca al Sol, en sus cagitas, baynas y pulpas del fruto sino es may jugosa, y despues 


\section{(4)}

\& colocan entre polvos de piedra pomez, gegun usan los Chínos para remitirlas de una parte, á otra; por que asi precave el que se enrancien, y por consiguiente se cona servan en actirud para propagarse. Toda especie de fruta particular, que se puede sesar entre arena, en la forma que queda prescripto para las flores singulares.

Las plantas parasitas, que son las que comunmente se erian, sobre los troncos de los arboles viejos, las Algas, Ongos \&c. que se encuentran sobre las vigas de parajes humedos en los maderos podridos de los monres, y en las peñas y piedras de las orillas de las aguas; las plantas maritimas que hecha el mar fuera, las que se hallar, prendidas en las rocas que bañan las aguas saladas, y las marinas, que se crian en las Playas y cercanias del mar, todas estas se ponen entre papeles bien extendidas, en la misma disposicion que dexo dicho de las demas plantas.

Los sucos de los vegerables, como son las leches de los balsamos, las resinas, las gomo resinas las resiras simples, las feculas, ò precipitados de algunas plantas tinctorias, $y$, las sales de algunas hiervas.

La hebra, ò hilaza que se saca de algunos vegetables, los vilanos de algunas plantas semejantes al Ailgodon, los aceytes de las Semillas, y arinas de rayzes tubsrosas, ò carnosas.

\section{DEL REINO ANIMAL.}

\footnotetext{
F Ste presenta en la Historia Natural el quadro mas delícioso, à los ojos del honbre, y de el se sacan tambien mas urilidades para la vida humara; pero como por lo general son tan suceptibles de corrupsion, los medios que se toman para conservarlos, de abrirlos, desollarlos salarlos \&c. los hace desfigurarse y perjer su toral merito y hermosura, y para que esto no suceda se dispondiàn en la forma siguiente.
} 


\section{(.5) \\ QUADKUPEDOS}

A Estos se les hace una incision iongitudinal desde cerea del ano, hasta el ombligo, por esta se và desprendiendo, el tegumento, ó piel, hasta la articulacion del femur, ó primer hueso del muslo con los innominados ò caderas: separado el de un lado se sigue á hacer lo mismo crn el otro, despues se busca la base de la cola y se tira de esta para ver si es tacil en desprenderse, sino se le hace una incision en toda su longitud para desollarla: despegadas estas tres extremidades, se sigue volviendo lo de dentro à fuera de la piel, hasta llegar â las escapulas; en este lugar se desunen las extremidades anteriores, por la articulacion, del humero, ó priner bueso del brazo, con la escapula. ò espaldilla; se siguse volviendo por el cuello y cabeza hasta la circunferencia de la voca, aqui se de ja toda la piel pren. did a a la cab:za, se despega esta de todo el cuerpo, por la primera vertebra que se une al occipital; por el agugero de este sele sacan los sesos, se descarna bien la calabera, los brazos y pies, hasta las palmas y plantas, conservandole rodos los huesos. Despues de bien descarnado, se empolva rodo con los polvos, de arsenico, azúre, ó qualquiera de las preparaciones de mercurio, y en falta de todos estos los de cebadilla, resinas olorosas, chilillo, rabaco en polvo \& $\mathrm{c}_{\text {. }}$ y antes que se seque se volbera cada parte a su lugar, con el pelo acia fuera; se rellenard la cola brazos, $y$ pies, de asèrin, arenilla ô salvado, en el cuerpo, un poco de estopa, o algodon, lo mismo para la voca, ojos y narice: va en este estado se pondra d secar, si la humejad del tiempo ó atmosfera del Pais lo retarda, se pondra dentro de un horno, con el calor mojecado, que solo seque y no tueste aunque se tare de un dia ò dos en engugarse.

\section{$A$ V ES}

1

Ogidas estas con tra npa, red, cervatana ò escopeta cor muricion muy monuda sin que se maltraten ni ensucit n las

Plu. 


\section{(6)}

Plumas y conserven su colocacion narural, lo que se eonedguirà manejandulas del pico y pies sin manosearlas. La disted cion se hace en esta forma, se le hace una inciion chlique del orificio del ano, a la vase de la ala de uno y otro laedo formando estas dos aberturas una $\mathrm{V}$, estas ircisiones interesaràn la base de la pierna por las quales se vuscarà la articulacion del femur, ó hueso del muslo con los innominados ó de las eaderas, para separar los pies del cuerpo, procurando que queden estos aderidos à la oja anterior, que se desprenderá con el cuvdado de no abrir la cabidad del vientre, para que no se ensucien las plumas con la sangre y humedad que suele salir de esra Rejion; lebantada esta oja anterior sobre las pechugas, se le pone un papel en lo humedo del cuero para que no se arrugue ni se peguen á el las Plumas: hecho esto se prosigue desprendien to la picl por la parte posterior en la misma forma, cn esta va unida la cola con el ultimo hueso del coccix ò $\mathrm{Rabz}$ dil a que sele deja al desprenderla de los demas, se prosigue vol víen do esta oja posterior, se le pone papel como à la anterior, y se descubre hata la escapula ò espaldilla, por la articulacion de esta con el humero, ó primer hueso de la ala se separa del cuerpo y queda inserta en la piel: se prosigue volviendo lo de adentro à fuera hasta la primera vertebra del cuello, por esta se desune de la cabesa, se le sacan los sesos, se limpian bien las alas, Irs pies y vase de la cola, y despues de bien empolvada la piel con los polvos de arsenico, azufre, mercurio \&c. se buelve cada parte à su lugar, se le pone un poco de algodon en el cuello, y en medio de la piel, y se pone à secar en la propia forma que los quadrupedos, sila armosfera, ò el Sol, no lo hace prontamente se mere en un horno pasado con el calor, que solo seque, y no rueste, $\nabla$ despues se guartan en caxon bien cerrado, con alcanfor, ì otro olor balsamico, para que se conserven hasra su remision.

\section{PECES.}

Os pescados pequeños que no pasan de dos libras se disea can del modo siguiente. Se da principio cortandole una porcioncita de curis aue tienen debaio lamandibula inferior ena 


\section{(7)}

Ire las dos agallas, echa esta incisiun se descubren blen estas, y sesacan enetas, y por el espacio que dejan se extraen las entrañas y la parte de carne y espinas que se pueda, que esto serd segun el manejo del quelo execura: en los prineros grados de corrupcion es quando está en mejor disposicion de hacerse esta maniobra y se extraen con mucha facilidad todas las carnes con una cuchara de plata, fierro ù otro metal, y: mucho mejor si se hacen de intento angostas y largas, ya descarnados, ò medio descarnados segun se hayan podido dejara se les introluce cantidad de los polvos que quedan prescriptos entre los intesticios de las carnes que le han quedado; en esta disposicion sin otra ninguna aberiura y in que le falte ninguna escama, por que de esras se cuida tanto en los pescados, como de las plumas en las Aves, se ponen á enjugar al bol, ó en el parage nas seco y deventilacion; por que no admiten el calor mas moderado del horno, sin arrugarse las ecamas, y por consiguiente se desfiguran mucho, aunque pasen a otros grados de corrupcion como no se rompa la piel con la potrciumbre antes de secarse, quedan en disposicion de poder os armar y embilsamar; en este estado se encaxonan, como queda dicho en las Aves, bien cerrados y con aicanfor, o balsamn. Si los pescados son muy grandes se les hace una abertura longirulinai desde el ano hasta la mirad del vientre, $\dot{o}$ en oonde sea mas tivinulad, que esro loha de diotarla fia gura del pez, la disposiciun delas aleras, y las circunstancias de su piel, si es de cuero ó escamas, por esra abertura se les sacan las entrafiasy porcion de carnes que sea posible como queda adverido para los demas; por las aburturas naturales, se les sacan las Agallas y ojos, y en todo lo restante se seguira el Eeginen prescripro,

\section{A MFIBIOS.}

$\mathrm{F}$

ctos son los Animales mas faciles de disecar: las culebras; Ranas, y algunos lagartos, ò lagartijas se les caca toda la car$n=y$ huesos por la voca en esta forma: se le abien bien las mannihulas, se les d sprend: del occipal la primera virtebra del cuello estirando de esta, la fluxibilidad del cuero, y car- 


\section{8}

we le hace volverse con facilivad lo de denrro a fuera ic to to el cuerpo; al liegar á las exirenicares st curtan cinoer los quadrupedos y se descarnan; bien limfio toro st destrurcllan todas las fartes bolviendolas a su situacion natural y pur la yuca se rellena, to angosto de la cola y éxtremidadts, conarenilla fina mezclada con polvos de arsenico, azufse \& c. y lo restante de serrin de maderas resinosas y atomaticas, y desputs se ponen à secar en la aetirud mas vatural que sea posible.

\section{INSECTOS.}

As mariposas, escarabajos y otros insectos pequeños no necesitan de disecacion; tstos por lo comun se crgen vivos, primero se les arraviesa por el cuerpo unalfiler de arriba abaxo, y se les hace morir dandoles porla voca con un pincel, sin ensuciar los colores de las alas, unas gotas de qualquiera de los azeyes so espiritus esenciales con lo que quedan muerros y embalsamado; despues se colocan en caxilas con los fondos de qualquiera rabla blanda, ó corchosa que se puedan clavar con facilidad los alfieres, y en defeeto de estas tablas se ponea en los fondos una capa de cera y resinas del canto de dos pea sos y sobre esta un papel para que no se peguen: asi se van colocando con el cuidado de que que len sus alas y pies abiertos principalnence en las mariposas y demas insefos de alas. Las conchas de agua dulce y salada se procuran adquirir recin entes antes que lascalcine el Sol, si puede ser cois el animal seco dentro, y con roda su incrustacion exterior que suele ser aspera y fia; las bivalvis se remiten hermanadas con sus compañea ras. Los caraccles en la misma forma con su tapa, ó comptierta, que por lo regular son huesusas, principalmenre en los maritimos, los herizos, estrellas y ortas producciones del mar", se secaná la sombra para que el sol no consuma los hermosos cor lores de que suelen estar marizadas.

Finalmente se colectará de quarto producen los rres Reys nos de la naturaleza, que no sea comun en todas partes. Sin olvidar alg nnos primores y rarezas del arte, y rambien los utensilios, armas, vestimentas, idolos, y quanto foseen y usa los Gentiles. 


\section{(9) ADVERIENCIAS.}

1

As producciones del Reyno, Vegetal y Mineral, despues de bien secas se enbuelven entre algudon, estopa, ù otra ma. teria blanda y elastica que ajuste todas las piezas en el caxon en que se han de conducir; pues de lo contratio se maltrata y desfigura todo, con los golpes que suelen sua frir en los caminus; ta nbien proporcionarán los medios mas equitativos en sus conduciones. Los pedazos de Piedras, ninerales, madreporas, y otras produciones que se crian en el mar, se ha de procurar, si las circunstancias lo permiten que sean lo menos, del tamaño de un puño cerrado, y quando meno: duplicadas, ò triplicadas, y si son de poco peso no importa que se multipliquen.

Despues de bien colozadas las pieles de los Animales en los terminos que queda dicho, dentro de caxones, se cerraràn las averturas y rendijas de esros, con cera, despues se embrean bien sabre crudo, ú otro lienzo, y si hay, proporcion en lugar del embreado se le dan muchas manos de leche de ule, hasta que quede con la consistencia necesaria, de modo que no penetre el aire, por consiguience la hamedad.

A Real Sociedad ofrece un premio de una Medalla de Oro de rres onzas, que costea el Nasuralista Don Joseph Longinos Martinez, al que remira mejor coleccion de producciones Naturales en el terroino de seis meses, que se conraràn, desde el dia de la fecha, y hara expresa, y honorifica mencion en sus actas publicas del $\bar{q}$ sea acredor al premio y de aquellos sujetos que haciento remesas para este $\mathrm{Ga}$ vinete acrediten mas sa zelo por la iastruccion y la urilidad comun.

\section{NOTA}

Los Cajones vendran dirigidos à la Real Sociedad, y dentro de estos vendra la instrucios, y lista de lo que concienen, y el nombre del sujeto, y al reconocerlos graduará el Varuealita el merito de cada uno para la ajudicacioa del premio.

Nueva Guatemala y Eenero \&. de 1797 . 



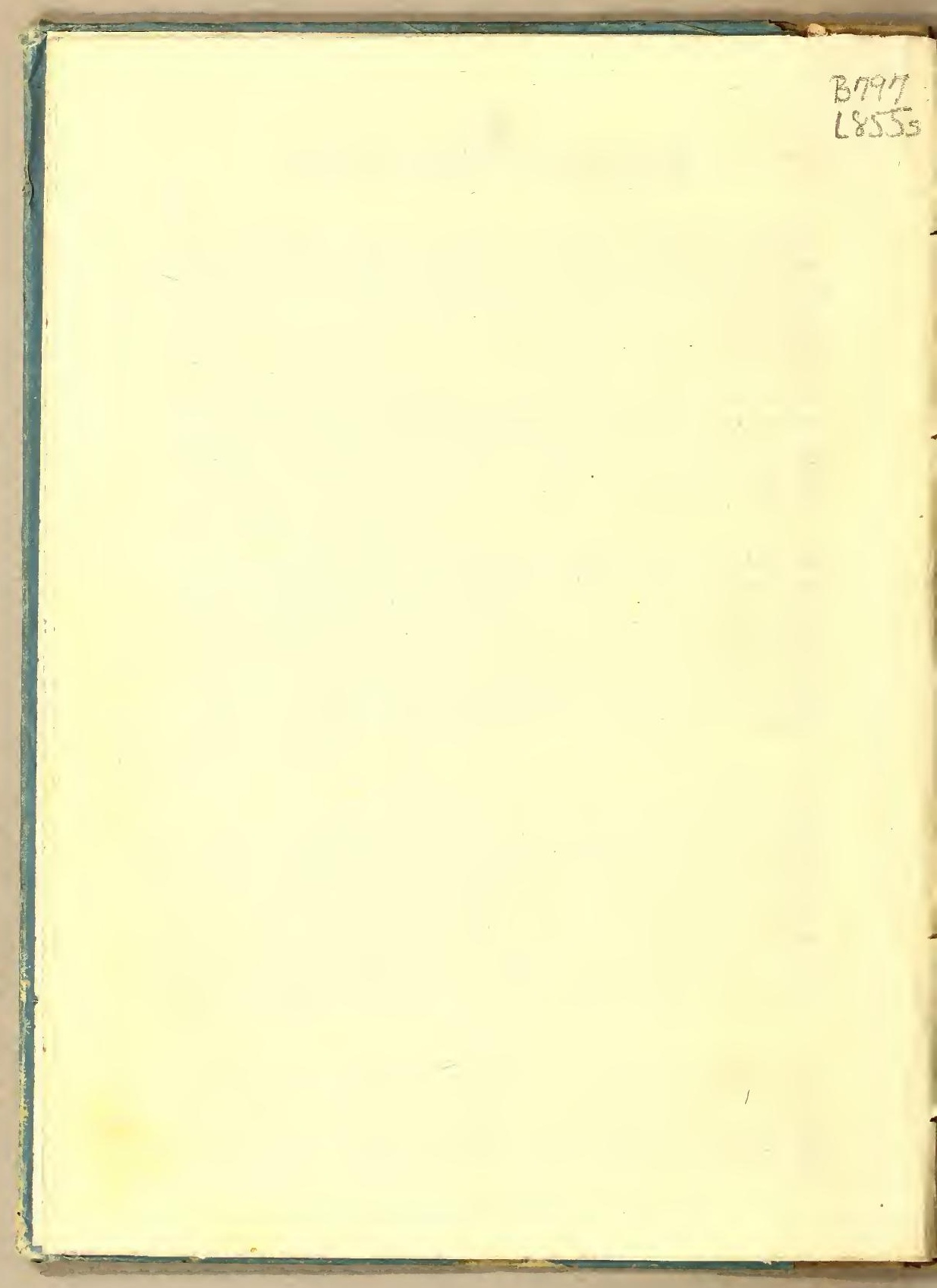




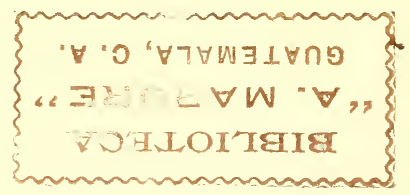




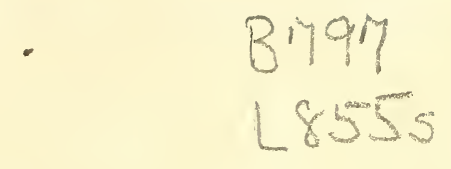




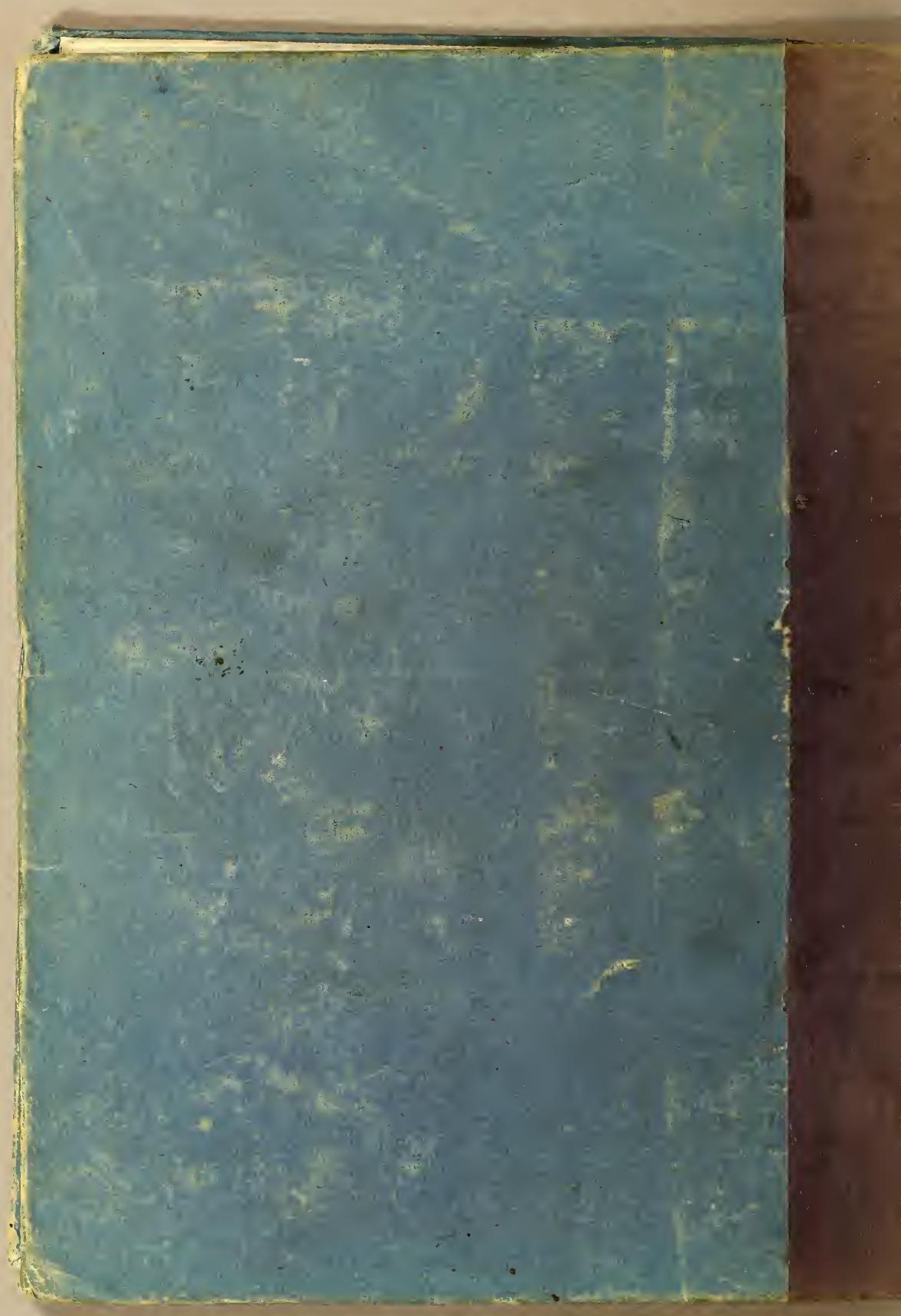

